\title{
Identification of an Asymmetric Pauson-Khand Precatalyst
}

\author{
Susan E. Gibson, ${ }^{*}{ }^{\dagger,}$ Sara E. Lewis, ${ }^{\dagger}$ Jennifer A. Loch, ${ }^{\dagger, \dagger}$ Jonathan W. Steed, ${ }^{\dagger}$ and \\ Matthew J. Tozer ${ }^{\S}$ \\ Department of Chemistry, King's College London, Strand, London WC2R 2LS, United Kingdom, \\ and Medivir UK, Peterhouse Technology Park, 100 Fulbourn Road, Cambridge \\ CB1 9PT, United Kingdom
}

The structure of 3 was solved and refined using the programs SHELXS- $97^{1}$ and SHELXL-97, ${ }^{2}$ respectively. The program X-Seed ${ }^{3}$ was used as an interface to the SHELX programs and to prepare the figures.

(1) Sheldrick, G. M. Acta Crystallogr., Sect. A 1990, 46, 467.

(2) Sheldrick, G. M. University of Göttingen, 1997.

(3) Barbour, L. J. J. Supramol. Chem. 2001, 1, 189. 
Table 1. Crystal data and structure refinement for 3 .

Identification code

Empirical formula

Formula weight

Temperature

Wavelength

Crystal system

Space group

Unit cell dimensions

Volume

Z

Density (calculated)

Absorption coefficient

$\mathrm{F}(000)$

Crystal size

Theta range for data collection

Index ranges

Reflections collected

Independent reflections

Completeness to theta $=25.00^{\circ}$

Absorption correction

Max. and min. transmission

Refinement method

Data / restraints / parameters

Goodness-of-fit on $\mathrm{F}^{2}$

Final $\mathrm{R}$ indices [I $>2 \operatorname{sigma}(\mathrm{I})]$

$\mathrm{R}$ indices (all data)

Extinction coefficient

Largest diff. peak and hole
C:.CIF

C51.50 H35 Cl3 Co2 O6 P2

1035.95

120(2) K

$0.71073 \AA$

Monoclinic

P21/n

$\mathrm{a}=14.4575(3) \AA$

$\alpha=90^{\circ}$.

$\mathrm{b}=19.3414(4) \AA$ $\beta=94.5540(10)^{\circ}$.

$c=16.2073(4) \AA$ $\gamma=90^{\circ}$.

4517.71(17) $\AA^{3}$

4

$1.523 \mathrm{Mg} / \mathrm{m}^{3}$

$1.034 \mathrm{~mm}^{-1}$

2108

$0.15 \times 0.10 \times 0.10 \mathrm{~mm}^{3}$

1.76 to $25.00^{\circ}$.

$-17<=\mathrm{h}<=17,-23<=\mathrm{k}<=22,-19<=1<=19$

14623

$7931[\mathrm{R}(\mathrm{int})=0.0779]$

$99.7 \%$

Semi-empirical from equivalents

0.9036 and 0.8603

Full-matrix least-squares on $\mathrm{F}^{2}$

7931 / 0 / 587

1.028

$\mathrm{R} 1=0.0582, \mathrm{wR} 2=0.1380$

$\mathrm{R} 1=0.1198, \mathrm{wR} 2=0.1608$

$0.0010(4)$

1.394 and -0.958 e. $\AA^{-3}$ 
Table 2. Atomic coordinates $\left(\mathrm{x} 10^{4}\right)$ and equivalent isotropic displacement parameters $\left(\AA^{2} \times 10^{3}\right)$ for 3. $U(e q)$ is defined as one third of the trace of the orthogonalized $U^{i j}$ tensor.

\begin{tabular}{|c|c|c|c|c|}
\hline & $\mathrm{x}$ & $\mathrm{y}$ & $\mathrm{z}$ & $\mathrm{U}(\mathrm{eq})$ \\
\hline $\operatorname{Co}(1)$ & $1535(1)$ & $1743(1)$ & $8355(1)$ & $20(1)$ \\
\hline $\operatorname{Co}(2)$ & $3201(1)$ & $1498(1)$ & $7968(1)$ & $35(1)$ \\
\hline $\mathrm{P}(1)$ & $726(1)$ & 1961(1) & 9433(1) & $18(1)$ \\
\hline $\mathrm{P}(2)$ & $523(1)$ & 2314(1) & $7460(1)$ & $19(1)$ \\
\hline $\mathrm{O}(1)$ & $1305(3)$ & $255(2)$ & $8663(2)$ & $42(1)$ \\
\hline $\mathrm{O}(2)$ & $2862(2)$ & $2769(2)$ & $9032(2)$ & $39(1)$ \\
\hline $\mathrm{O}(3)$ & $1765(3)$ & $1219(2)$ & $6666(2)$ & $39(1)$ \\
\hline $\mathrm{O}(4)$ & 4344(3) & 1497(3) & $9560(3)$ & $73(2)$ \\
\hline $\mathrm{O}(5)$ & $3708(3)$ & $138(2)$ & $7322(3)$ & $61(1)$ \\
\hline $\mathrm{O}(6)$ & $4080(3)$ & 2611(2) & 7073(3) & $56(1)$ \\
\hline$C(1)$ & $1363(3)$ & $840(3)$ & $8525(3)$ & $29(1)$ \\
\hline$C(2)$ & 2446(3) & 2312(3) & 8709(3) & $30(1)$ \\
\hline$C(3)$ & 2091(4) & 1414(3) & 7318(4) & $30(1)$ \\
\hline$C(4)$ & $3916(4)$ & $1478(4)$ & $8944(5)$ & $51(2)$ \\
\hline$C(5)$ & $3505(4)$ & $661(4)$ & 7598(4) & $45(2)$ \\
\hline$C(6)$ & 3713(4) & 2175(3) & $7420(4)$ & $42(2)$ \\
\hline$C(7)$ & $-442(3)$ & $1643(2)$ & $9127(3)$ & $17(1)$ \\
\hline$C(8)$ & $-641(3)$ & $936(2)$ & 9273(3) & $20(1)$ \\
\hline$C(9)$ & $-1471(3)$ & $654(3)$ & $8977(3)$ & $24(1)$ \\
\hline$C(10)$ & $-2151(3)$ & $1053(2)$ & $8510(3)$ & $21(1)$ \\
\hline $\mathrm{C}(11)$ & $-3024(3)$ & $777(3)$ & $8236(3)$ & $29(1)$ \\
\hline$C(12)$ & $-3675(4)$ & 1170(3) & 7797(3) & $32(1)$ \\
\hline$C(13)$ & $-3470(3)$ & 1859(3) & 7602(3) & $28(1)$ \\
\hline$C(14)$ & $-2632(3)$ & 2141(3) & 7858(3) & $23(1)$ \\
\hline$C(15)$ & $-1949(3)$ & $1748(2)$ & $8332(3)$ & $18(1)$ \\
\hline$C(16)$ & $-1071(3)$ & $2047(2)$ & $8645(3)$ & $19(1)$ \\
\hline$C(17)$ & $-877(3)$ & $2788(2)$ & 8441(3) & $18(1)$ \\
\hline$C(18)$ & $-1408(3)$ & $3306(2)$ & $8817(3)$ & $19(1)$ \\
\hline$C(19)$ & $-2019(3)$ & $3137(3)$ & 9431(3) & $23(1)$ \\
\hline$C(20)$ & $-2504(3)$ & $3651(3)$ & 9801(3) & $28(1)$ \\
\hline $\mathrm{C}(21)$ & $-2424(3)$ & 4344(3) & 9559(3) & $26(1)$ \\
\hline
\end{tabular}




\begin{tabular}{|c|c|c|c|c|}
\hline $\mathrm{C}(22)$ & $-1854(3)$ & $4514(3)$ & 8959(3) & $26(1)$ \\
\hline $\mathrm{C}(23)$ & $-1317(3)$ & $4009(2)$ & $8588(3)$ & $19(1)$ \\
\hline $\mathrm{C}(24)$ & $-684(3)$ & $4177(3)$ & 7999(3) & $21(1)$ \\
\hline$C(25)$ & $-156(3)$ & $3680(2)$ & 7673(3) & $21(1)$ \\
\hline$C(26)$ & $-234(3)$ & $2974(2)$ & 7885(3) & $17(1)$ \\
\hline$C(27)$ & 1084(3) & $1513(2)$ & $10404(3)$ & $21(1)$ \\
\hline$C(28)$ & $471(4)$ & $1318(3)$ & 10980(3) & $25(1)$ \\
\hline$C(29)$ & $805(4)$ & 1079(3) & $11750(3)$ & $31(1)$ \\
\hline$C(30)$ & 1745(4) & $1023(3)$ & 11966(3) & $34(1)$ \\
\hline $\mathrm{C}(31)$ & $2348(4)$ & $1202(3)$ & $11386(3)$ & $32(1)$ \\
\hline$C(32)$ & 2036(4) & $1435(3)$ & $10610(3)$ & $27(1)$ \\
\hline$C(33)$ & $571(3)$ & $2835(2)$ & $9825(3)$ & $19(1)$ \\
\hline$C(34)$ & $9(3)$ & $2937(3)$ & $10476(3)$ & $26(1)$ \\
\hline$C(35)$ & $-137(4)$ & $3593(3)$ & $10776(3)$ & $31(1)$ \\
\hline$C(36)$ & $290(4)$ & $4164(3)$ & $10443(3)$ & $29(1)$ \\
\hline $\mathrm{C}(37)$ & $855(3)$ & $4069(3)$ & $9802(3)$ & $28(1)$ \\
\hline$C(38)$ & $996(3)$ & $3417(3)$ & $9500(3)$ & $23(1)$ \\
\hline C(39) & $-358(3)$ & $1820(2)$ & 6844(3) & $18(1)$ \\
\hline$C(40)$ & $-410(4)$ & $1108(3)$ & 6888(3) & $27(1)$ \\
\hline$C(41)$ & $-1144(4)$ & $744(3)$ & 6486(3) & $30(1)$ \\
\hline$C(42)$ & $-1839(4)$ & 1111(3) & 6033(3) & $32(1)$ \\
\hline$C(43)$ & $-1802(3)$ & $1814(3)$ & 5975(3) & $27(1)$ \\
\hline$C(44)$ & $-1069(3)$ & $2170(3)$ & $6376(3)$ & $24(1)$ \\
\hline $\mathrm{C}(45)$ & 1134(3) & $2806(3)$ & $6700(3)$ & $23(1)$ \\
\hline$C(46)$ & $970(3)$ & $2727(3)$ & $5846(3)$ & $27(1)$ \\
\hline$C(47)$ & 1498(4) & $3105(3)$ & $5318(3)$ & $36(1)$ \\
\hline $\mathrm{C}(48)$ & 2163(4) & $3550(3)$ & $5623(4)$ & $38(2)$ \\
\hline$C(49)$ & 2332(4) & $3642(3)$ & 6463(4) & $39(2)$ \\
\hline$C(50)$ & $1829(4)$ & $3263(3)$ & 7003(3) & $31(1)$ \\
\hline $\mathrm{Cl}(1 \mathrm{~S})$ & $5253(1)$ & $3328(1)$ & $8823(1)$ & $78(1)$ \\
\hline $\mathrm{Cl}(2 \mathrm{~S})$ & 4111(2) & $4552(1)$ & $8933(2)$ & 131(1) \\
\hline $\mathrm{Cl}(3 \mathrm{~S})$ & 4934(2) & $205(1)$ & $10857(1)$ & $79(1)$ \\
\hline$C(1 S)$ & $4733(7)$ & $3868(4)$ & $9429(5)$ & $88(3)$ \\
\hline $\mathrm{C}(2 \mathrm{~S})$ & $4389(10)$ & $-149(7)$ & $9999(8)$ & $62(4)$ \\
\hline
\end{tabular}


Table 3. Bond lengths $[\AA]$ and angles $\left[^{\circ}\right]$ for 3 .

\begin{tabular}{|c|c|}
\hline $\mathrm{Co}(1)-\mathrm{C}(2)$ & $1.777(6)$ \\
\hline $\mathrm{Co}(1)-\mathrm{C}(1)$ & $1.788(6)$ \\
\hline $\mathrm{Co}(1)-\mathrm{C}(3)$ & $2.021(5)$ \\
\hline $\mathrm{Co}(1)-\mathrm{P}(1)$ & $2.2196(14)$ \\
\hline $\mathrm{Co}(1)-\mathrm{P}(2)$ & $2.2653(14)$ \\
\hline $\mathrm{Co}(1)-\mathrm{Co}(2)$ & $2.5801(9)$ \\
\hline $\operatorname{Co}(2)-C(6)$ & $1.775(7)$ \\
\hline $\mathrm{Co}(2)-\mathrm{C}(5)$ & $1.793(7)$ \\
\hline $\mathrm{Co}(2)-\mathrm{C}(4)$ & $1.820(7)$ \\
\hline $\mathrm{Co}(2)-\mathrm{C}(3)$ & $1.855(6)$ \\
\hline $\mathrm{Co}(2)-\mathrm{C}(2)$ & $2.307(5)$ \\
\hline $\mathrm{P}(1)-\mathrm{C}(33)$ & $1.826(5)$ \\
\hline $\mathrm{P}(1)-\mathrm{C}(7)$ & $1.828(5)$ \\
\hline $\mathrm{P}(1)-\mathrm{C}(27)$ & $1.835(5)$ \\
\hline $\mathrm{P}(2)-\mathrm{C}(39)$ & $1.825(5)$ \\
\hline $\mathrm{P}(2)-\mathrm{C}(45)$ & $1.838(5)$ \\
\hline $\mathrm{P}(2)-\mathrm{C}(26)$ & $1.850(5)$ \\
\hline $\mathrm{O}(1)-\mathrm{C}(1)$ & $1.157(6)$ \\
\hline $\mathrm{O}(2)-\mathrm{C}(2)$ & $1.169(6)$ \\
\hline $\mathrm{O}(3)-\mathrm{C}(3)$ & $1.184(6)$ \\
\hline $\mathrm{O}(4)-\mathrm{C}(4)$ & $1.133(7)$ \\
\hline $\mathrm{O}(5)-\mathrm{C}(5)$ & $1.154(7)$ \\
\hline $\mathrm{O}(6)-\mathrm{C}(6)$ & $1.165(7)$ \\
\hline$C(7)-C(16)$ & $1.390(6)$ \\
\hline$C(7)-C(8)$ & $1.422(6)$ \\
\hline$C(8)-C(9)$ & $1.369(6)$ \\
\hline $\mathrm{C}(9)-\mathrm{C}(10)$ & $1.419(7)$ \\
\hline$C(10)-C(11)$ & $1.410(7)$ \\
\hline$C(10)-C(15)$ & $1.410(7)$ \\
\hline $\mathrm{C}(11)-\mathrm{C}(12)$ & $1.365(7)$ \\
\hline$C(12)-C(13)$ & $1.406(7)$ \\
\hline$C(13)-C(14)$ & $1.363(7)$ \\
\hline$C(14)-C(15)$ & $1.422(6)$ \\
\hline$C(15)-C(16)$ & $1.450(6)$ \\
\hline
\end{tabular}




\begin{tabular}{|c|c|}
\hline$C(16)-C(17)$ & $1.502(7)$ \\
\hline$C(17)-C(26)$ & $1.392(6)$ \\
\hline $\mathrm{C}(17)-\mathrm{C}(18)$ & $1.428(7)$ \\
\hline $\mathrm{C}(18)-\mathrm{C}(23)$ & $1.419(7)$ \\
\hline $\mathrm{C}(18)-\mathrm{C}(19)$ & $1.420(7)$ \\
\hline$C(19)-C(20)$ & $1.381(7)$ \\
\hline$C(20)-C(21)$ & $1.402(7)$ \\
\hline$C(21)-C(22)$ & $1.364(7)$ \\
\hline$C(22)-C(23)$ & $1.412(7)$ \\
\hline$C(23)-C(24)$ & $1.412(6)$ \\
\hline$C(24)-C(25)$ & $1.359(7)$ \\
\hline$C(25)-C(26)$ & $1.415(6)$ \\
\hline $\mathrm{C}(27)-\mathrm{C}(28)$ & $1.389(7)$ \\
\hline $\mathrm{C}(27)-\mathrm{C}(32)$ & $1.399(7)$ \\
\hline $\mathrm{C}(28)-\mathrm{C}(29)$ & $1.382(7)$ \\
\hline $\mathrm{C}(29)-\mathrm{C}(30)$ & $1.381(7)$ \\
\hline $\mathrm{C}(30)-\mathrm{C}(31)$ & $1.375(7)$ \\
\hline $\mathrm{C}(31)-\mathrm{C}(32)$ & $1.377(7)$ \\
\hline $\mathrm{C}(33)-\mathrm{C}(34)$ & $1.396(7)$ \\
\hline $\mathrm{C}(33)-\mathrm{C}(38)$ & $1.404(7)$ \\
\hline $\mathrm{C}(34)-\mathrm{C}(35)$ & $1.382(7)$ \\
\hline$C(35)-C(36)$ & $1.395(7)$ \\
\hline $\mathrm{C}(36)-\mathrm{C}(37)$ & $1.383(7)$ \\
\hline $\mathrm{C}(37)-\mathrm{C}(38)$ & $1.374(7)$ \\
\hline$C(39)-C(40)$ & $1.382(7)$ \\
\hline $\mathrm{C}(39)-\mathrm{C}(44)$ & $1.402(6)$ \\
\hline $\mathrm{C}(40)-\mathrm{C}(41)$ & $1.392(7)$ \\
\hline $\mathrm{C}(41)-\mathrm{C}(42)$ & $1.391(7)$ \\
\hline $\mathrm{C}(42)-\mathrm{C}(43)$ & $1.364(7)$ \\
\hline $\mathrm{C}(43)-\mathrm{C}(44)$ & $1.382(7)$ \\
\hline $\mathrm{C}(45)-\mathrm{C}(46)$ & $1.394(7)$ \\
\hline $\mathrm{C}(45)-\mathrm{C}(50)$ & $1.398(7)$ \\
\hline $\mathrm{C}(46)-\mathrm{C}(47)$ & $1.398(7)$ \\
\hline $\mathrm{C}(47)-\mathrm{C}(48)$ & $1.354(8)$ \\
\hline $\mathrm{C}(48)-\mathrm{C}(49)$ & $1.375(8)$ \\
\hline$C(49)-C(50)$ & $1.390(7)$ \\
\hline
\end{tabular}




\begin{tabular}{|c|c|}
\hline Cl(1S)-C(1S) & $1.654(9)$ \\
\hline $\mathrm{Cl}(2 \mathrm{~S})-\mathrm{C}(1 \mathrm{~S})$ & $1.758(8)$ \\
\hline $\mathrm{Cl}(3 \mathrm{~S})-\mathrm{C}(2 \mathrm{~S})$ & $1.688(13)$ \\
\hline $\mathrm{Cl}(3 \mathrm{~S})-\mathrm{C}(2 \mathrm{~S}) \# 1$ & $1.764(14)$ \\
\hline C(2S)-Cl(3S)\#1 & $1.764(14)$ \\
\hline $\mathrm{C}(2)-\mathrm{Co}(1)-\mathrm{C}(1)$ & $131.6(2)$ \\
\hline $\mathrm{C}(2)-\mathrm{Co}(1)-\mathrm{C}(3)$ & $97.5(2)$ \\
\hline $\mathrm{C}(1)-\mathrm{Co}(1)-\mathrm{C}(3)$ & $83.7(2)$ \\
\hline $\mathrm{C}(2)-\mathrm{Co}(1)-\mathrm{P}(1)$ & $92.92(17)$ \\
\hline $\mathrm{C}(1)-\mathrm{Co}(1)-\mathrm{P}(1)$ & $88.60(16)$ \\
\hline $\mathrm{C}(3)-\mathrm{Co}(1)-\mathrm{P}(1)$ & $169.53(17)$ \\
\hline $\mathrm{C}(2)-\mathrm{Co}(1)-\mathrm{P}(2)$ & $109.23(18)$ \\
\hline $\mathrm{C}(1)-\mathrm{Co}(1)-\mathrm{P}(2)$ & $118.97(17)$ \\
\hline $\mathrm{C}(3)-\mathrm{Co}(1)-\mathrm{P}(2)$ & $84.33(16)$ \\
\hline $\mathrm{P}(1)-\mathrm{Co}(1)-\mathrm{P}(2)$ & $93.24(5)$ \\
\hline $\mathrm{C}(2)-\mathrm{Co}(1)-\mathrm{Co}(2)$ & $60.65(17)$ \\
\hline $\mathrm{C}(1)-\mathrm{Co}(1)-\mathrm{Co}(2)$ & $90.18(16)$ \\
\hline $\mathrm{C}(3)-\mathrm{Co}(1)-\mathrm{Co}(2)$ & $45.59(16)$ \\
\hline $\mathrm{P}(1)-\mathrm{Co}(1)-\mathrm{Co}(2)$ & $141.90(5)$ \\
\hline $\mathrm{P}(2)-\mathrm{Co}(1)-\mathrm{Co}(2)$ & $120.15(4)$ \\
\hline $\mathrm{C}(6)-\mathrm{Co}(2)-\mathrm{C}(5)$ & $112.1(3)$ \\
\hline $\mathrm{C}(6)-\mathrm{Co}(2)-\mathrm{C}(4)$ & $102.7(3)$ \\
\hline $\mathrm{C}(5)-\mathrm{Co}(2)-\mathrm{C}(4)$ & $97.7(3)$ \\
\hline $\mathrm{C}(6)-\mathrm{Co}(2)-\mathrm{C}(3)$ & $99.1(3)$ \\
\hline $\mathrm{C}(5)-\mathrm{Co}(2)-\mathrm{C}(3)$ & $87.4(2)$ \\
\hline $\mathrm{C}(4)-\mathrm{Co}(2)-\mathrm{C}(3)$ & $153.8(3)$ \\
\hline $\mathrm{C}(6)-\mathrm{Co}(2)-\mathrm{C}(2)$ & $89.5(2)$ \\
\hline $\mathrm{C}(5)-\mathrm{Co}(2)-\mathrm{C}(2)$ & $158.2(2)$ \\
\hline $\mathrm{C}(4)-\mathrm{Co}(2)-\mathrm{C}(2)$ & $79.7(2)$ \\
\hline $\mathrm{C}(3)-\mathrm{Co}(2)-\mathrm{C}(2)$ & $86.1(2)$ \\
\hline $\mathrm{C}(6)-\mathrm{Co}(2)-\mathrm{Co}(1)$ & $114.88(19)$ \\
\hline $\mathrm{C}(5)-\mathrm{Co}(2)-\mathrm{Co}(1)$ & $120.29(19)$ \\
\hline $\mathrm{C}(4)-\mathrm{Co}(2)-\mathrm{Co}(1)$ & $105.6(2)$ \\
\hline $\mathrm{C}(3)-\mathrm{Co}(2)-\mathrm{Co}(1)$ & $51.08(17)$ \\
\hline $\mathrm{C}(2)-\mathrm{Co}(2)-\mathrm{Co}(1)$ & $42.19(13)$ \\
\hline
\end{tabular}




\begin{tabular}{|c|c|}
\hline $\mathrm{C}(33)-\mathrm{P}(1)-\mathrm{C}(7)$ & $105.6(2)$ \\
\hline $\mathrm{C}(33)-\mathrm{P}(1)-\mathrm{C}(27)$ & $99.9(2)$ \\
\hline C(7)-P(1)-C(27) & $105.4(2)$ \\
\hline $\mathrm{C}(33)-\mathrm{P}(1)-\mathrm{Co}(1)$ & $122.48(16)$ \\
\hline $\mathrm{C}(7)-\mathrm{P}(1)-\mathrm{Co}(1)$ & $104.83(15)$ \\
\hline $\mathrm{C}(27)-\mathrm{P}(1)-\mathrm{Co}(1)$ & $117.08(17)$ \\
\hline C(39)-P(2)-C(45) & $104.9(2)$ \\
\hline$C(39)-P(2)-C(26)$ & $99.1(2)$ \\
\hline $\mathrm{C}(45)-\mathrm{P}(2)-\mathrm{C}(26)$ & $102.9(2)$ \\
\hline $\mathrm{C}(39)-\mathrm{P}(2)-\mathrm{Co}(1)$ & $118.79(16)$ \\
\hline $\mathrm{C}(45)-\mathrm{P}(2)-\mathrm{Co}(1)$ & $111.14(16)$ \\
\hline $\mathrm{C}(26)-\mathrm{P}(2)-\mathrm{Co}(1)$ & $118.03(15)$ \\
\hline $\mathrm{O}(1)-\mathrm{C}(1)-\mathrm{Co}(1)$ & $175.6(5)$ \\
\hline $\mathrm{O}(2)-\mathrm{C}(2)-\mathrm{Co}(1)$ & $162.7(5)$ \\
\hline $\mathrm{O}(2)-\mathrm{C}(2)-\mathrm{Co}(2)$ & $120.1(4)$ \\
\hline $\mathrm{Co}(1)-\mathrm{C}(2)-\mathrm{Co}(2)$ & $77.2(2)$ \\
\hline $\mathrm{O}(3)-\mathrm{C}(3)-\mathrm{Co}(2)$ & $143.0(4)$ \\
\hline $\mathrm{O}(3)-\mathrm{C}(3)-\mathrm{Co}(1)$ & $133.3(4)$ \\
\hline $\mathrm{Co}(2)-\mathrm{C}(3)-\mathrm{Co}(1)$ & $83.3(2)$ \\
\hline $\mathrm{O}(4)-\mathrm{C}(4)-\mathrm{Co}(2)$ & $176.6(6)$ \\
\hline $\mathrm{O}(5)-\mathrm{C}(5)-\mathrm{Co}(2)$ & $176.4(6)$ \\
\hline $\mathrm{O}(6)-\mathrm{C}(6)-\mathrm{Co}(2)$ & $177.5(6)$ \\
\hline $\mathrm{C}(16)-\mathrm{C}(7)-\mathrm{C}(8)$ & $120.1(4)$ \\
\hline$C(16)-C(7)-P(1)$ & $120.9(4)$ \\
\hline $\mathrm{C}(8)-\mathrm{C}(7)-\mathrm{P}(1)$ & $118.2(3)$ \\
\hline $\mathrm{C}(9)-\mathrm{C}(8)-\mathrm{C}(7)$ & $120.5(4)$ \\
\hline C(8)-C(9)-C(10) & $121.4(5)$ \\
\hline $\mathrm{C}(11)-\mathrm{C}(10)-\mathrm{C}(15)$ & $119.4(4)$ \\
\hline $\mathrm{C}(11)-\mathrm{C}(10)-\mathrm{C}(9)$ & $121.8(4)$ \\
\hline $\mathrm{C}(15)-\mathrm{C}(10)-\mathrm{C}(9)$ & $118.7(4)$ \\
\hline $\mathrm{C}(12)-\mathrm{C}(11)-\mathrm{C}(10)$ & $121.1(5)$ \\
\hline $\mathrm{C}(11)-\mathrm{C}(12)-\mathrm{C}(13)$ & $119.8(5)$ \\
\hline $\mathrm{C}(14)-\mathrm{C}(13)-\mathrm{C}(12)$ & $120.6(5)$ \\
\hline$C(13)-C(14)-C(15)$ & $120.9(5)$ \\
\hline$C(10)-C(15)-C(14)$ & $118.3(4)$ \\
\hline $\mathrm{C}(10)-\mathrm{C}(15)-\mathrm{C}(16)$ & $119.9(4)$ \\
\hline
\end{tabular}




\begin{tabular}{|c|c|}
\hline$C(14)-C(15)-C(16)$ & $121.8(4)$ \\
\hline$C(7)-C(16)-C(15)$ & $119.2(4)$ \\
\hline$C(7)-C(16)-C(17)$ & $122.3(4)$ \\
\hline $\mathrm{C}(15)-\mathrm{C}(16)-\mathrm{C}(17)$ & $118.5(4)$ \\
\hline$C(26)-C(17)-C(18)$ & $120.2(4)$ \\
\hline$C(26)-C(17)-C(16)$ & $122.3(4)$ \\
\hline$C(18)-C(17)-C(16)$ & $117.4(4)$ \\
\hline$C(23)-C(18)-C(19)$ & $118.7(4)$ \\
\hline$C(23)-C(18)-C(17)$ & $119.8(4)$ \\
\hline $\mathrm{C}(19)-\mathrm{C}(18)-\mathrm{C}(17)$ & $121.5(4)$ \\
\hline$C(20)-C(19)-C(18)$ & $120.3(5)$ \\
\hline$C(19)-C(20)-C(21)$ & $120.7(5)$ \\
\hline$C(22)-C(21)-C(20)$ & $119.8(5)$ \\
\hline $\mathrm{C}(21)-\mathrm{C}(22)-\mathrm{C}(23)$ & $121.4(5)$ \\
\hline$C(24)-C(23)-C(22)$ & $122.5(5)$ \\
\hline $\mathrm{C}(24)-\mathrm{C}(23)-\mathrm{C}(18)$ & $118.4(4)$ \\
\hline$C(22)-C(23)-C(18)$ & $119.0(4)$ \\
\hline$C(25)-C(24)-C(23)$ & $121.0(5)$ \\
\hline $\mathrm{C}(24)-\mathrm{C}(25)-\mathrm{C}(26)$ & $121.9(4)$ \\
\hline$C(17)-C(26)-C(25)$ & $118.5(4)$ \\
\hline $\mathrm{C}(17)-\mathrm{C}(26)-\mathrm{P}(2)$ & $120.5(4)$ \\
\hline $\mathrm{C}(25)-\mathrm{C}(26)-\mathrm{P}(2)$ & $120.9(3)$ \\
\hline $\mathrm{C}(28)-\mathrm{C}(27)-\mathrm{C}(32)$ & $118.6(4)$ \\
\hline $\mathrm{C}(28)-\mathrm{C}(27)-\mathrm{P}(1)$ & $123.6(4)$ \\
\hline $\mathrm{C}(32)-\mathrm{C}(27)-\mathrm{P}(1)$ & $117.5(4)$ \\
\hline $\mathrm{C}(29)-\mathrm{C}(28)-\mathrm{C}(27)$ & $120.1(5)$ \\
\hline $\mathrm{C}(30)-\mathrm{C}(29)-\mathrm{C}(28)$ & $121.4(5)$ \\
\hline $\mathrm{C}(31)-\mathrm{C}(30)-\mathrm{C}(29)$ & $118.2(5)$ \\
\hline $\mathrm{C}(30)-\mathrm{C}(31)-\mathrm{C}(32)$ & $121.7(5)$ \\
\hline $\mathrm{C}(31)-\mathrm{C}(32)-\mathrm{C}(27)$ & $119.9(5)$ \\
\hline $\mathrm{C}(34)-\mathrm{C}(33)-\mathrm{C}(38)$ & $117.9(5)$ \\
\hline $\mathrm{C}(34)-\mathrm{C}(33)-\mathrm{P}(1)$ & $119.2(4)$ \\
\hline $\mathrm{C}(38)-\mathrm{C}(33)-\mathrm{P}(1)$ & $122.8(4)$ \\
\hline $\mathrm{C}(35)-\mathrm{C}(34)-\mathrm{C}(33)$ & $120.7(5)$ \\
\hline $\mathrm{C}(34)-\mathrm{C}(35)-\mathrm{C}(36)$ & $120.4(5)$ \\
\hline$C(37)-C(36)-C(35)$ & $119.4(5)$ \\
\hline
\end{tabular}




$\begin{array}{lr}\mathrm{C}(38)-\mathrm{C}(37)-\mathrm{C}(36) & 120.2(5) \\ \mathrm{C}(37)-\mathrm{C}(38)-\mathrm{C}(33) & 121.3(5) \\ \mathrm{C}(40)-\mathrm{C}(39)-\mathrm{C}(44) & 117.9(4) \\ \mathrm{C}(40)-\mathrm{C}(39)-\mathrm{P}(2) & 122.2(4) \\ \mathrm{C}(44)-\mathrm{C}(39)-\mathrm{P}(2) & 119.5(4) \\ \mathrm{C}(39)-\mathrm{C}(40)-\mathrm{C}(41) & 121.5(5) \\ \mathrm{C}(42)-\mathrm{C}(41)-\mathrm{C}(40) & 118.7(5) \\ \mathrm{C}(43)-\mathrm{C}(42)-\mathrm{C}(41) & 121.1(5) \\ \mathrm{C}(42)-\mathrm{C}(43)-\mathrm{C}(44) & 119.7(5) \\ \mathrm{C}(43)-\mathrm{C}(44)-\mathrm{C}(39) & 121.1(5) \\ \mathrm{C}(46)-\mathrm{C}(45)-\mathrm{C}(50) & 118.7(5) \\ \mathrm{C}(46)-\mathrm{C}(45)-\mathrm{P}(2) & 123.7(4) \\ \mathrm{C}(50)-\mathrm{C}(45)-\mathrm{P}(2) & 117.5(4) \\ \mathrm{C}(45)-\mathrm{C}(46)-\mathrm{C}(47) & 119.5(5) \\ \mathrm{C}(48)-\mathrm{C}(47)-\mathrm{C}(46) & 121.0(5) \\ \mathrm{C}(47)-\mathrm{C}(48)-\mathrm{C}(49) & 120.5(5) \\ \mathrm{C}(48)-\mathrm{C}(49)-\mathrm{C}(50) & 119.7(5) \\ \mathrm{C}(49)-\mathrm{C}(50)-\mathrm{C}(45) & 120.6(5) \\ \mathrm{C}(2 \mathrm{~S})-\mathrm{Cl}(3 \mathrm{~S})-\mathrm{C}(2 \mathrm{~S}) \# 1 & 65.1(8) \\ \mathrm{Cl}(1 \mathrm{~S})-\mathrm{C}(1 \mathrm{~S})-\mathrm{Cl}(2 \mathrm{~S}) & 116.4(5) \\ \mathrm{Cl}(3 \mathrm{~S})-\mathrm{C}(2 \mathrm{~S})-\mathrm{Cl}(3 \mathrm{~S}) \# 1 & 114.9(8)\end{array}$

Symmetry transformations used to generate equivalent atoms:

$\# 1-\mathrm{x}+1,-\mathrm{y},-\mathrm{z}+2$ 
Table 4. Anisotropic displacement parameters $\left(\AA^{2} \times 10^{3}\right)$ for 3. The anisotropic displacement factor exponent takes the form: $-2 \pi^{2}\left[h^{2} a^{* 2} U^{11}+\ldots+2 h k a^{*} b^{*} U^{12}\right]$

\begin{tabular}{|c|c|c|c|c|c|c|}
\hline & $\mathrm{U}^{11}$ & $\mathrm{U}^{22}$ & $\mathrm{U}^{33}$ & $\mathrm{U}^{23}$ & $\mathrm{U}^{13}$ & $\mathrm{U}^{12}$ \\
\hline $\operatorname{Co}(1)$ & $20(1)$ & $20(1)$ & $20(1)$ & $-1(1)$ & $1(1)$ & $2(1)$ \\
\hline $\operatorname{Co}(2)$ & $27(1)$ & $37(1)$ & $42(1)$ & $-1(1)$ & $5(1)$ & $3(1)$ \\
\hline $\mathrm{P}(1)$ & $20(1)$ & $17(1)$ & $18(1)$ & $-1(1)$ & $0(1)$ & $0(1)$ \\
\hline $\mathrm{P}(2)$ & $20(1)$ & $19(1)$ & $18(1)$ & $-1(1)$ & 1(1) & $-1(1)$ \\
\hline $\mathrm{O}(1)$ & $54(3)$ & $22(2)$ & $50(3)$ & $4(2)$ & $15(2)$ & $8(2)$ \\
\hline $\mathrm{O}(2)$ & $30(2)$ & $49(3)$ & $38(2)$ & $-14(2)$ & $8(2)$ & $-9(2)$ \\
\hline $\mathrm{O}(3)$ & $47(2)$ & $41(2)$ & $29(2)$ & $-7(2)$ & $4(2)$ & $7(2)$ \\
\hline $\mathrm{O}(4)$ & $63(3)$ & $82(4)$ & $67(4)$ & $-1(3)$ & $-29(3)$ & $14(3)$ \\
\hline $\mathrm{O}(5)$ & $54(3)$ & $43(3)$ & $86(4)$ & $-13(3)$ & $5(3)$ & $25(2)$ \\
\hline $\mathrm{O}(6)$ & $53(3)$ & $56(3)$ & $63(3)$ & $-6(2)$ & $28(2)$ & $-11(2)$ \\
\hline $\mathrm{C}(1)$ & $32(3)$ & $36(4)$ & 21(3) & $-4(3)$ & $8(2)$ & $10(3)$ \\
\hline$C(2)$ & $22(3)$ & $37(3)$ & $31(3)$ & $-5(3)$ & $5(2)$ & $-3(3)$ \\
\hline$C(3)$ & $33(3)$ & $26(3)$ & $32(3)$ & $5(3)$ & $7(3)$ & $9(3)$ \\
\hline$C(4)$ & $35(4)$ & $52(4)$ & $65(5)$ & $1(4)$ & $0(3)$ & $9(3)$ \\
\hline$C(5)$ & $27(3)$ & $52(4)$ & $54(4)$ & $4(3)$ & $-3(3)$ & 11(3) \\
\hline$C(6)$ & $35(3)$ & $51(4)$ & $41(4)$ & $-7(3)$ & $13(3)$ & $-7(3)$ \\
\hline$C(7)$ & $18(2)$ & $19(3)$ & $15(3)$ & $-1(2)$ & $3(2)$ & $-1(2)$ \\
\hline$C(8)$ & $21(3)$ & $20(3)$ & $20(3)$ & $-2(2)$ & $1(2)$ & $0(2)$ \\
\hline$C(9)$ & $26(3)$ & $19(3)$ & $27(3)$ & $-1(2)$ & $6(2)$ & $-1(2)$ \\
\hline$C(10)$ & $23(3)$ & $21(3)$ & $18(3)$ & $-3(2)$ & $2(2)$ & $-1(2)$ \\
\hline $\mathrm{C}(11)$ & $27(3)$ & $26(3)$ & $34(3)$ & $-1(2)$ & $-2(2)$ & $-11(3)$ \\
\hline$C(12)$ & $21(3)$ & $37(3)$ & $35(3)$ & $-2(3)$ & $-10(2)$ & $-11(3)$ \\
\hline$C(13)$ & $24(3)$ & $30(3)$ & $28(3)$ & $-1(2)$ & $-7(2)$ & $-2(2)$ \\
\hline$C(14)$ & $27(3)$ & $19(3)$ & $24(3)$ & $0(2)$ & $1(2)$ & $-2(2)$ \\
\hline$C(15)$ & $16(2)$ & $19(3)$ & $18(3)$ & $-1(2)$ & $2(2)$ & $0(2)$ \\
\hline$C(16)$ & $20(3)$ & $18(3)$ & $18(3)$ & $-4(2)$ & $5(2)$ & $0(2)$ \\
\hline$C(17)$ & $16(2)$ & $20(3)$ & $16(3)$ & $-3(2)$ & $-6(2)$ & $1(2)$ \\
\hline$C(18)$ & $17(2)$ & $19(3)$ & $20(3)$ & $1(2)$ & $-5(2)$ & $-1(2)$ \\
\hline$C(19)$ & $21(3)$ & $22(3)$ & $26(3)$ & $2(2)$ & $0(2)$ & $1(2)$ \\
\hline$C(20)$ & $25(3)$ & $31(3)$ & $27(3)$ & $1(2)$ & $1(2)$ & $3(2)$ \\
\hline $\mathrm{C}(21)$ & $23(3)$ & $26(3)$ & $27(3)$ & $-9(2)$ & $-1(2)$ & $3(2)$ \\
\hline
\end{tabular}




\begin{tabular}{|c|c|c|c|c|c|c|}
\hline$C(22)$ & $27(3)$ & $20(3)$ & $32(3)$ & $-7(2)$ & $-4(2)$ & $0(2)$ \\
\hline$C(23)$ & $19(3)$ & $21(3)$ & $17(3)$ & $-3(2)$ & $-5(2)$ & $-1(2)$ \\
\hline$C(24)$ & $25(3)$ & $14(3)$ & $23(3)$ & $2(2)$ & $-5(2)$ & $-3(2)$ \\
\hline$C(25)$ & $21(3)$ & $22(3)$ & $19(3)$ & $1(2)$ & $-3(2)$ & $-2(2)$ \\
\hline$C(26)$ & $18(2)$ & $18(3)$ & $16(3)$ & $-3(2)$ & $-3(2)$ & $-4(2)$ \\
\hline $\mathrm{C}(27)$ & $29(3)$ & $15(3)$ & $16(3)$ & $-3(2)$ & $-8(2)$ & $2(2)$ \\
\hline $\mathrm{C}(28)$ & $29(3)$ & $21(3)$ & $26(3)$ & $-2(2)$ & $4(2)$ & $4(2)$ \\
\hline$C(29)$ & $51(4)$ & $20(3)$ & $22(3)$ & $0(2)$ & $2(3)$ & $0(3)$ \\
\hline$C(30)$ & $52(4)$ & $19(3)$ & $26(3)$ & $0(2)$ & $-15(3)$ & $-3(3)$ \\
\hline$C(31)$ & $33(3)$ & $20(3)$ & $38(4)$ & $4(3)$ & $-14(3)$ & $-2(2)$ \\
\hline$C(32)$ & $28(3)$ & $17(3)$ & $34(3)$ & $1(2)$ & $-5(2)$ & $-6(2)$ \\
\hline$C(33)$ & $20(3)$ & $21(3)$ & $16(3)$ & $-1(2)$ & $-7(2)$ & $3(2)$ \\
\hline$C(34)$ & $35(3)$ & $14(3)$ & $26(3)$ & $0(2)$ & $-6(2)$ & $2(2)$ \\
\hline$C(35)$ & $38(3)$ & $34(3)$ & $21(3)$ & $-8(3)$ & $-1(2)$ & $6(3)$ \\
\hline$C(36)$ & $36(3)$ & $18(3)$ & $32(3)$ & $-6(2)$ & $-10(3)$ & $2(2)$ \\
\hline$C(37)$ & $32(3)$ & $14(3)$ & $36(3)$ & $2(2)$ & $-10(3)$ & $-5(2)$ \\
\hline$C(38)$ & $18(3)$ & $26(3)$ & $26(3)$ & $-3(2)$ & $-2(2)$ & $-1(2)$ \\
\hline C(39) & $18(2)$ & $21(3)$ & $15(3)$ & $-2(2)$ & $2(2)$ & $2(2)$ \\
\hline$C(40)$ & $33(3)$ & $23(3)$ & $25(3)$ & $6(2)$ & $0(2)$ & $1(2)$ \\
\hline$C(41)$ & $41(3)$ & $21(3)$ & $28(3)$ & $1(2)$ & $-5(3)$ & $-12(3)$ \\
\hline$C(42)$ & $33(3)$ & $35(4)$ & $27(3)$ & $1(3)$ & $-5(3)$ & $-12(3)$ \\
\hline$C(43)$ & $27(3)$ & $35(3)$ & $17(3)$ & $-2(2)$ & $-2(2)$ & $4(3)$ \\
\hline $\mathrm{C}(44)$ & $25(3)$ & $27(3)$ & $19(3)$ & $-1(2)$ & $2(2)$ & $-3(2)$ \\
\hline$C(45)$ & $21(3)$ & $21(3)$ & $26(3)$ & $4(2)$ & $6(2)$ & $4(2)$ \\
\hline $\mathrm{C}(46)$ & $32(3)$ & $25(3)$ & $26(3)$ & $0(2)$ & $7(2)$ & $6(2)$ \\
\hline$C(47)$ & $40(3)$ & $43(4)$ & $26(3)$ & $0(3)$ & $12(3)$ & $7(3)$ \\
\hline $\mathrm{C}(48)$ & $41(3)$ & $38(4)$ & $38(4)$ & $9(3)$ & $19(3)$ & $0(3)$ \\
\hline$C(49)$ & $31(3)$ & $33(4)$ & $54(4)$ & $5(3)$ & $10(3)$ & $-8(3)$ \\
\hline $\mathrm{C}(50)$ & $32(3)$ & $33(3)$ & $28(3)$ & $5(3)$ & $5(2)$ & $-2(3)$ \\
\hline $\mathrm{Cl}(1 \mathrm{~S})$ & $59(1)$ & $122(2)$ & $53(1)$ & $11(1)$ & $5(1)$ & $-1(1)$ \\
\hline $\mathrm{Cl}(2 \mathrm{~S})$ & $157(3)$ & $91(2)$ & $153(3)$ & $49(2)$ & $66(2)$ & $24(2)$ \\
\hline $\mathrm{Cl}(3 \mathrm{~S})$ & $111(2)$ & $79(2)$ & $49(1)$ & $-4(1)$ & $12(1)$ & $5(1)$ \\
\hline$C(1 S)$ & $142(8)$ & $56(5)$ & $63(5)$ & $11(4)$ & $-2(5)$ & $1(5)$ \\
\hline$C(2 S)$ & $79(10)$ & $54(9)$ & $50(9)$ & $0(7)$ & $-8(8)$ & $-13(8)$ \\
\hline
\end{tabular}


Table 5. Hydrogen coordinates $\left(\mathrm{x} 10^{4}\right)$ and isotropic displacement parameters $\left(\AA^{2} \times 10^{3}\right)$ for 3 .

\begin{tabular}{|c|c|c|c|c|}
\hline & $\mathrm{x}$ & $\mathrm{y}$ & $\mathrm{z}$ & $\mathrm{U}(\mathrm{eq})$ \\
\hline $\mathrm{H}(8)$ & -195 & 656 & 9578 & 24 \\
\hline $\mathrm{H}(9)$ & -1595 & 182 & 9087 & 29 \\
\hline $\mathrm{H}(11)$ & -3162 & 309 & 8358 & 35 \\
\hline $\mathrm{H}(12)$ & -4263 & 978 & 7623 & 38 \\
\hline $\mathrm{H}(13)$ & -3921 & 2130 & 7291 & 34 \\
\hline $\mathrm{H}(14)$ & -2502 & 2605 & 7717 & 28 \\
\hline $\mathrm{H}(19)$ & -2094 & 2669 & 9588 & 28 \\
\hline $\mathrm{H}(20)$ & -2896 & 3535 & 10224 & 33 \\
\hline $\mathrm{H}(21)$ & -2766 & 4693 & 9813 & 31 \\
\hline $\mathrm{H}(22)$ & -1818 & 4982 & 8788 & 32 \\
\hline $\mathrm{H}(24)$ & -626 & 4643 & 7828 & 25 \\
\hline $\mathrm{H}(25)$ & 278 & 3811 & 7291 & 25 \\
\hline $\mathrm{H}(28)$ & -179 & 1348 & 10844 & 30 \\
\hline $\mathrm{H}(29)$ & 378 & 950 & 12140 & 37 \\
\hline $\mathrm{H}(30)$ & 1970 & 865 & 12499 & 40 \\
\hline $\mathrm{H}(31)$ & 2997 & 1164 & 11525 & 38 \\
\hline $\mathrm{H}(32)$ & 2467 & 1543 & 10216 & 32 \\
\hline $\mathrm{H}(34)$ & -275 & 2551 & 10716 & 31 \\
\hline $\mathrm{H}(35)$ & -531 & 3656 & 11212 & 37 \\
\hline $\mathrm{H}(36)$ & 195 & 4614 & 10654 & 35 \\
\hline $\mathrm{H}(37)$ & 1146 & 4455 & 9570 & 33 \\
\hline $\mathrm{H}(38)$ & 1389 & 3358 & 9062 & 28 \\
\hline $\mathrm{H}(40)$ & 66 & 861 & 7201 & 32 \\
\hline $\mathrm{H}(41)$ & -1170 & 254 & 6519 & 36 \\
\hline $\mathrm{H}(42)$ & -2347 & 869 & 5761 & 38 \\
\hline $\mathrm{H}(43)$ & -2278 & 2058 & 5659 & 32 \\
\hline $\mathrm{H}(44)$ & -1047 & 2659 & 6334 & 28 \\
\hline $\mathrm{H}(46)$ & 503 & 2419 & 5625 & 33 \\
\hline $\mathrm{H}(47)$ & 1389 & 3048 & 4736 & 43 \\
\hline $\mathrm{H}(48)$ & 2516 & 3800 & 5254 & 46 \\
\hline
\end{tabular}




\begin{tabular}{lrrrr}
$\mathrm{H}(49)$ & 2790 & 3962 & 6672 & 47 \\
$\mathrm{H}(50)$ & 1959 & 3316 & 7584 & 37 \\
$\mathrm{H}(1 \mathrm{~S} 1)$ & 5210 & 4067 & 9832 & 105 \\
$\mathrm{H}(1 \mathrm{~S} 2)$ & 4300 & 3598 & 9747 & 105 \\
$\mathrm{H}(2 \mathrm{~S} 1)$ & 3829 & 128 & 9836 & 74 \\
$\mathrm{H}(2 \mathrm{~S} 2)$ & 4181 & -620 & 10136 & 74 \\
\hline
\end{tabular}

